\title{
Economic Assessment of Russian Nuclear Strategies on the Basis of Fast Breeder Reactors
}

\author{
O. V. Marchenko and S. V. Solomin \\ Energy Systems Institute of Russian Academy of Sciences, 130 Lermontov Street, Irkutsk 664033, Russia \\ Correspondence should be addressed to O. V. Marchenko; marchenko@isem.sei.irk.ru
}

Received 28 March 2013; Revised 26 August 2013; Accepted 6 September 2013

Academic Editor: Michael F. Simpson

Copyright (C) 2013 O. V. Marchenko and S. V. Solomin. This is an open access article distributed under the Creative Commons Attribution License, which permits unrestricted use, distribution, and reproduction in any medium, provided the original work is properly cited.

\begin{abstract}
The paper assesses the economic risk caused by the delay in commissioning innovative nuclear power plants with fast breeder reactors in Russia. The risk is quantitatively measured by the excessive costs for energy development and the possibility of implementing the considered variants that differ in power consumption, technical and economic indices of the reactors, and constraints on $\mathrm{CO}_{2}$ emissions. The probability distribution functions of economic losses for different strategies of nuclear energy development are constructed.
\end{abstract}

\section{Introduction}

Investigation of long-term perspectives of world development shows that nuclear energy is the most efficient means for decreasing $\mathrm{CO}_{2}$ emissions [1]. Fast breeder reactors (FBR) should be applied also due to depletion of economically efficient uranium-235 resources [1-5]. The closed fuel cycle will allow using the additional fissile material, that is, plutonium received from uranium-238 isotope $(99.3 \%$ in natural uranium), while now only natural fissile material-uranium$235(0.7 \%$ in natural uranium), is used. Thus it will allow increasing the nuclear fuel reserves that will be sufficient throughout thousands of years.

In February 2010 the Government of Russia approved the Federal Target Program until 2020 designed to bring a new technology platform for the nuclear power industry based on fast reactors with a closed fuel cycle [6]. The paper aims to assess the economic risk caused by the delay in the large-scale commissioning of nuclear power plants with fast breeder reactors in Russia.

In order to consider the relations of different energy sources among themselves and with the environment the energy sector should be treated as a whole system. The paper considers the nuclear power industry as part of the energy sector in Russia that, in turn, is analyzed in background of the world energy sector development. This is justified by the fact that the cost of energy resources in Russia, including uranium (and correspondingly the fuel component of energy cost), is determined not only by the costs for their production but also by the world market prices.

\section{Method}

Future conditions of energy development are characterized by high uncertainty that usually is considered on the basis of a scenario approach [7-14]. We formulated two scenarios of constraints on $\mathrm{CO}_{2}$ emissions (soft and strict), three scenarios of power consumption (low, medium, and high), and four scenarios of technological development of the nuclear energy, which in total makes up 24 calculation variants. The calculations were performed using the global energy model (GEM) [1, 12-14].

The GEM model belongs to a class of mathematical models that are based on the "bottom-up" approach and is intended for determination of an optimal technological structure of the world energy system with specified energy demands. The model describes the world energy system as a set of interconnected processes of primary energy resources extraction or production, their transformation into secondary energy carriers and final energy. In the model the world energy system is represented by several interconnected 
regional energy systems with distinguishing the following regions: North America, Europe, Japan and Korea, Australia and New Zealand, Russia, countries of the former Soviet Union, Latin America, Middle East and North Africa, the rest of Africa, China, and South and Southeast Asia.

The GEM model sequentially optimises the regional energy structure and the interregional fuel exchange for the years 2025, 2050, 2075, and 2100 with further transfer of the obtained structure as an initial one for the coming 25-year period.

The model contains a description of several hundred energy technologies, including two types of nuclear power plants: power plants with thermal neutron reactors that use uranium-235 as a fuel and power plants with fast neutron reactors (breeders) that use uranium-238 (as an additive to the main fuel-plutonium).

Each technology $j$ for the region $r$ is characterised by the specific costs on product output $-c_{r j}$, the specific consumption of resources and the outputs of products and harmful substances, and the constraints on minimum and maximum scales of utilisation. The yearly production of technologies $\left(x_{r j}\right)$ is the optimised variables of the model.

The specific costs for each type of technological installations were calculated by the formula

$$
c_{r j}=\left(a_{k} K_{r j}+S_{r j}\right) \frac{\gamma}{h_{r j}},
$$

where

$$
a_{k}=\frac{p}{\left[1-(1+p)^{-T_{r j}}\right]} .
$$

Here $K_{r j}$ represent specific investments, US\$/kW; $S_{r j}$ represent specific constant operating costs, US\$/(kW/year); $p$ represent a discount rate, $1 /$ year; $T_{r j}$ represent service life of technological installation, years, $h_{r j}$ represent annual number of utilisation hours, $\gamma=278 \mathrm{~kW} / \mathrm{h} / \mathrm{GJ}$.

The objective function of the model is the discounted total expenditures (investments and operating costs) on regional energy systems (for the whole technological chain) and the interregional transport of energy carriers for the 25-year period. The technological structure of the world energy system can be represented mathematically in the following form: determine the minimum of the objective function:

$$
z=\sum_{r} \sum_{j} c_{r j} x_{r j}
$$

subject to technological, economic, and environmental constraints described in detail in the works $[1,14]$.

The scenarios of technological development differ in the time of commissioning fast breeder reactors, which is modeled by the specified constraints. We consider the following scenarios for commissioning fast breeder reactors: (1) the basic scenario that implies large-scale development of fast breeder reactors under the most favorable conditions; (2) "Scenario-20" that implies a delay in commissioning fast breeder reactors of approximately 20 years as compared to the basic scenario, (3) "Scenario-40"-a 40-year delay; (4) scenario "without FBR."

The fast breeder reactors have the best technical and economic indices in the basic scenario $(2700 \$ / \mathrm{kW}$ in 2025 and $2500 \$ / \mathrm{kW}$ in 2100 (in present-day dollars (US dollars for economic assessment is used through the article))) and the worst indices in "Scenario-40" (4400\$/kW and $4000 \$ / \mathrm{kW}$, resp.). Technical and economic indices of nuclear power plants and levels of power consumption were varied only for Russia. For the other regions of the world these data were taken as the average or most probable set of values.

We impose two types of constraints on $\mathrm{CO}_{2}$ emissions: soft and strict ones. Based on the scenarios of sustainable development $[1,11,13]$, we set limits on $\mathrm{CO}_{2}$ emissions for the world as a whole (33-50 Gt in 2050 and 30-55 Gt in 2100) (the higher value corresponds to the scenario with soft environmental constraints, and the smaller value to the scenario with strict environmental constraints). The limits for Russia are 2-2.5 Gt until 2050 and 1.8-2 Gt until 2100.

\section{Results and Discussion}

Optimization calculations of the technological structure of the energy sector in the world and in Russia until 2100 were made for all the formed variants on the GEM. The scale of nuclear energy development in Russia that is determined as a result of optimization is presented in Table 1 (the lower values of the capacity range correspond to the soft constraints on $\mathrm{CO}_{2}$ emissions, the higher values - to the strict constraints). In all the variants the construction of nuclear power plants with fast breeder reactors is economically efficient.

The delay in commissioning fast breeder reactors that is modeled by the constraints and the degradation of their technical and economic indices increases the costs for energy development (Table 2). This is explained by the fact that more efficient nuclear power plants with fast breeder reactors are replaced by nuclear power plants with thermal reactors (because of the switch to more and more expensive uranium, especially in the second half of the 21st century), hydrogen power plants, or renewable energy sources required to reduce $\mathrm{CO}_{2}$ emissions.

The results of mathematical modeling are applied to determine the economic losses caused by delay in breeder reactor commissioning. The unified monetary value of each variant is obtained by addition of the costs (the cost difference) to the corresponding discount factors and their reduction to one time period (2012), taking account of extra expenditures for research, design efforts, and infrastructure creation (nuclear fuel cycle plants).

Possibility of implementing the variants was estimated based on the subjective probabilities. We assumed that the world and Russian energy would develop to a great extent based on the past trends. Therefore, low power consumption is more probable than medium and high ones (their probability is 50,30 , and $20 \%$, resp.), and soft emission constraints are more probable than strict ones (their probability is 80 and $20 \%)$. 
TABLE 1: Scales of nuclear energy development (installed capacity) in Russia, GW.

\begin{tabular}{|c|c|c|c|c|c|c|}
\hline \multirow{3}{*}{ Years } & \multicolumn{6}{|c|}{ Scenarios } \\
\hline & \multicolumn{2}{|c|}{ Basic } & \multicolumn{2}{|c|}{ "Scenario-20" } & \multicolumn{2}{|c|}{ "Scenario-40" } \\
\hline & $\mathrm{TR}^{*}$ & $\mathrm{FBR}^{* *}$ & TR & FBR & TR & FBR \\
\hline \multicolumn{7}{|c|}{ Low power consumption } \\
\hline 2025 & $28-31$ & $1-7$ & $28-31$ & 0 & $28-31$ & 0 \\
\hline 2050 & $45-50$ & $21-23$ & $45-50$ & $8-10$ & $45-50$ & 0 \\
\hline 2075 & $30-35$ & $55-57$ & $30-35$ & $33-35$ & $30-35$ & $7-10$ \\
\hline 2100 & $8-10$ & $100-109$ & $8-10$ & $64-70$ & $8-10$ & $24-26$ \\
\hline \multicolumn{7}{|c|}{ Medium power consumption } \\
\hline 2025 & $32-34$ & $9-11$ & $32-34$ & 0 & $32-34$ & 0 \\
\hline 2050 & $70-75$ & $28-31$ & $70-75$ & $15-18$ & $70-75$ & 0 \\
\hline 2075 & $35-41$ & $70-77$ & $35-41$ & $55-60$ & $35-41$ & $15-21$ \\
\hline 2100 & $15-19$ & $115-133$ & $15-19$ & $90-102$ & $15-19$ & $45-53$ \\
\hline \multicolumn{7}{|c|}{ High power consumption } \\
\hline 2025 & $35-38$ & $15-16$ & $35-38$ & 0 & $35-38$ & 0 \\
\hline 2050 & $75-82$ & $50-54$ & $75-82$ & $25-30$ & $75-82$ & 0 \\
\hline 2075 & $45-53$ & $105-121$ & $45-53$ & $65-71$ & $45-53$ & $20-26$ \\
\hline 2100 & $20-30$ & $140-170$ & $20-30$ & $100-127$ & $20-30$ & $55-60$ \\
\hline
\end{tabular}

${ }^{*}$ Thermal reactors, ${ }^{* *}$ fast breeder reactors.

TABLE 2: Additional costs for energy development relative to the basic scenario, billion dollars per year.

\begin{tabular}{|c|c|c|c|}
\hline \multirow{2}{*}{ Years } & \multicolumn{3}{|c|}{ Scenarios } \\
\hline & "Scenario-20" & "Scenario-40" & "Without FBR" \\
\hline \multicolumn{4}{|c|}{ Low power consumption } \\
\hline 2025 & $0.2-3$ & $0.2-3$ & $0.2-3$ \\
\hline 2050 & $2-5$ & $3-8$ & $3-8$ \\
\hline 2075 & $10-12$ & $21-22$ & $25-26$ \\
\hline 2100 & $17-19$ & $34-36$ & $42-45$ \\
\hline \multicolumn{4}{|c|}{ Medium power consumption } \\
\hline 2025 & $1-6$ & $1-6$ & $1-6$ \\
\hline 2050 & $3-7$ & $11-16$ & $11-16$ \\
\hline 2075 & $10-11$ & $27-28$ & $35-36$ \\
\hline 2100 & $16-17$ & $35-39$ & $52-57$ \\
\hline \multicolumn{4}{|c|}{ High power consumption } \\
\hline 2025 & $6-11$ & $6-11$ & $6-11$ \\
\hline 2050 & $10-11$ & $20-21$ & $20-21$ \\
\hline 2075 & $25-26$ & $48-49$ & 58-59 \\
\hline 2100 & $20-21$ & $47-48$ & $65-66$ \\
\hline
\end{tabular}

We constructed the probability distribution functions of economic losses (probability that the loss will be no less than the specified value) for different strategies of nuclear energy development on the basis of the indicated data (Figure 1).

\section{Conclusion}

The obtained results allow the following conclusions to be drawn:

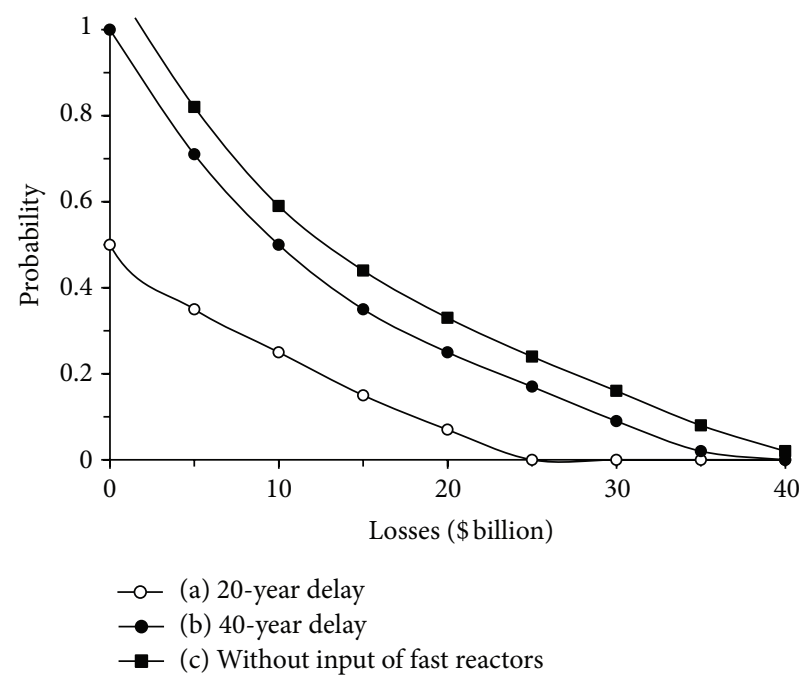

Figure 1: Probability distribution of the economic losses caused by delay in scaled commissioning of breeder reactors: (a) "Scenario-20",

(b) "Scenario-40", and (c): Scenario "without FBR".

(i) in Russia the commissioning of innovative nuclear power plants with breeder reactors is rational (earlier or later) for all the considered variants;

(ii) the higher is the power consumption and the stricter is the environmental constraints, the larger is the economic loss caused by delay in commissioning nuclear power plants with breeder reactors;

(iii) 20-year delay in commissioning nuclear power plants with breeder reactors is reasonable only in the case of expected low power consumption and absence of constraints on $\mathrm{CO}_{2}$ emissions; 
(iv) 20-year delay in commissioning nuclear power plants with breeder reactors with a probability of $50 \%$ will result in the loss that will be no less than 10 billion dollars with a probability of $25 \%$;

(v) delay in commissioning nuclear power plants with breeder reactors of 40 years and more will result in the loss of 10-12 billion dollars with a probability of $50 \%$;

(vi) complete refusal to develop nuclear power plants with breeder reactors in Russia will cause the economic loss up to 40 billion dollars.

\section{References}

[1] L. S. Belyaev, S. P. Filippov, O. V. Marchenko, S. V. Solomin, T. B. Stepanova, and A. L. Kokorinet, World Energy and Transition to Sustainable Development, Kluwer Academic, London, UK, 2002.

[2] C.-P. Zaleski, "Overview of future development of fast neutron reactors," Energy, vol. 23, no. 7-8, pp. 571-579, 1998.

[3] A. V. Zrodnikov, "Fast reactors in the energy security for the stable development of Russia," Atomic Energy, vol. 108, no. 4, pp. 230-233, 2010.

[4] E. O. Adamov, A. V. Dzhalavyan, A. V. Lopatkin et al., "Conceptual framework of a strategy for the development of nuclear power in Russia to 2100," Atomic Energy, vol. 112, no. 6, pp. 391403, 2012.

[5] A. Bharadwaj, S. Rajgopal, L. V. Krishnan, and K. S. Bellarmine, Economic of Fast Breeder Reactors. Indian Scenario, Center for Study of Science, Technology and Policy, Bangalore, India, 2012.

[6] Federal Target Program, "Nuclear power technologies of a new generation for 2010-2015 and till 2020," Order of the Government of the Russian Federation, No. 50, Moscow, Russia, February 2010, (Russian).

[7] N. Nakicenovic, A. Grübler, M. Jefferson et al., Global Energy Perspectives to 2050 and Beyond, International Institute for Applied Systems Analysis, World Energy Council, London, UK, 1995.

[8] N. Nakicenovic, A. Grübler, A. McDonald et al., Global Energy Perspectives, Cambridge University Press, Cambridge, UK, 1998.

[9] A. Grübler A, N. Nakicenovic, and D. G. Victor, "Dynamics of energy technologies and global change," Energy Policy, vol. 27, no. 5, pp. 247-280, 1999.

[10] C. P. Zaleski, Ed., Scenarios of Nuclear Power Growth in the 21st Century, Centre of Geopolitics of Energy and Raw Materials, Univercity of Paris IX Dauphine, Paris, France, 2002.

[11] V. V. Bushuev and A. M. Mastepanov, Eds., Global Energy \& Sustainable Development (White Book), International Sustainable Energy Development Centre, Moscow, Russia, 2009.

[12] L. S. Belyaev, O. V. Marchenko, S. P. Filippov, and S. V. Solomin, "Studies on competitiveness of space and terrestrial solar power plants using global energy model," International Journal of Global Energy Issues, vol. 25, no. 1-2, pp. 94-108, 2006.

[13] L. S. Belyaev, O. V. Marchenko, and S. V. Solomin, "A study on long-term trends in energy development in Russia and the world," Thermal Engineering, vol. 58, no. 13, pp. 1087-1093, 2011.

[14] L. S. Belyaev, O. V. Marchenko, and S. V. Solomin, "A study of wind energy contribution to global climate change mitigation," International Journal of Energy Technology and Policy, vol. 3, no. 4, pp. 324-341, 2005. 


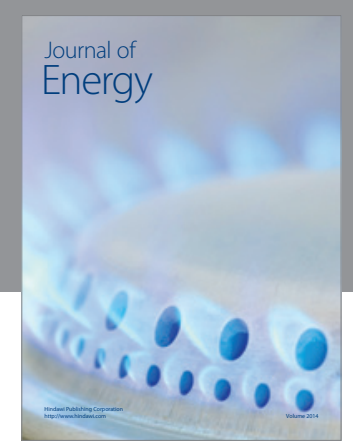

Journal of

Industrial Engineering
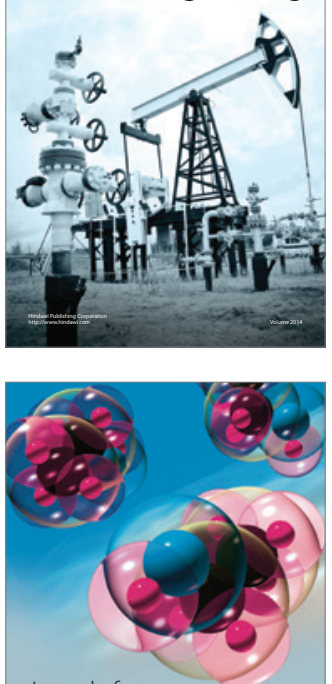

Fuels
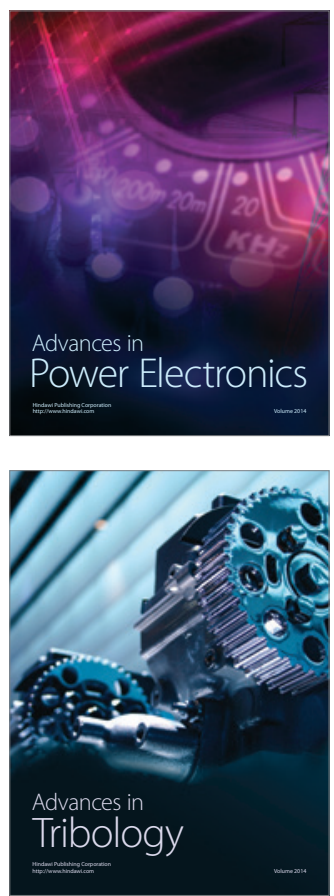

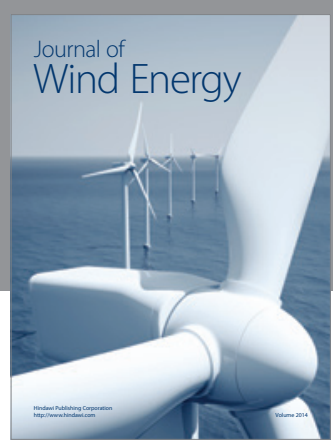

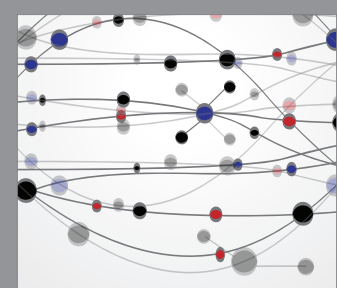

The Scientific World Journal

Submit your manuscripts at http://www.hindawi.com

Journal of

Structures
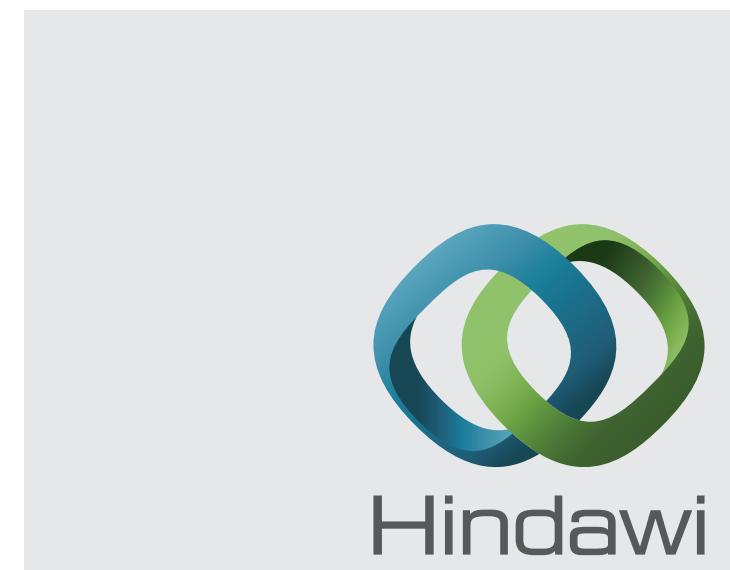

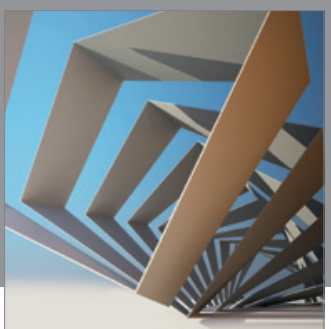

Rotating

Machinery
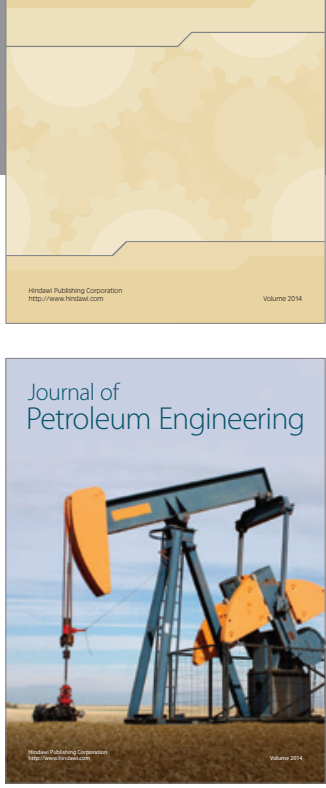

Journal of

Solar Energy
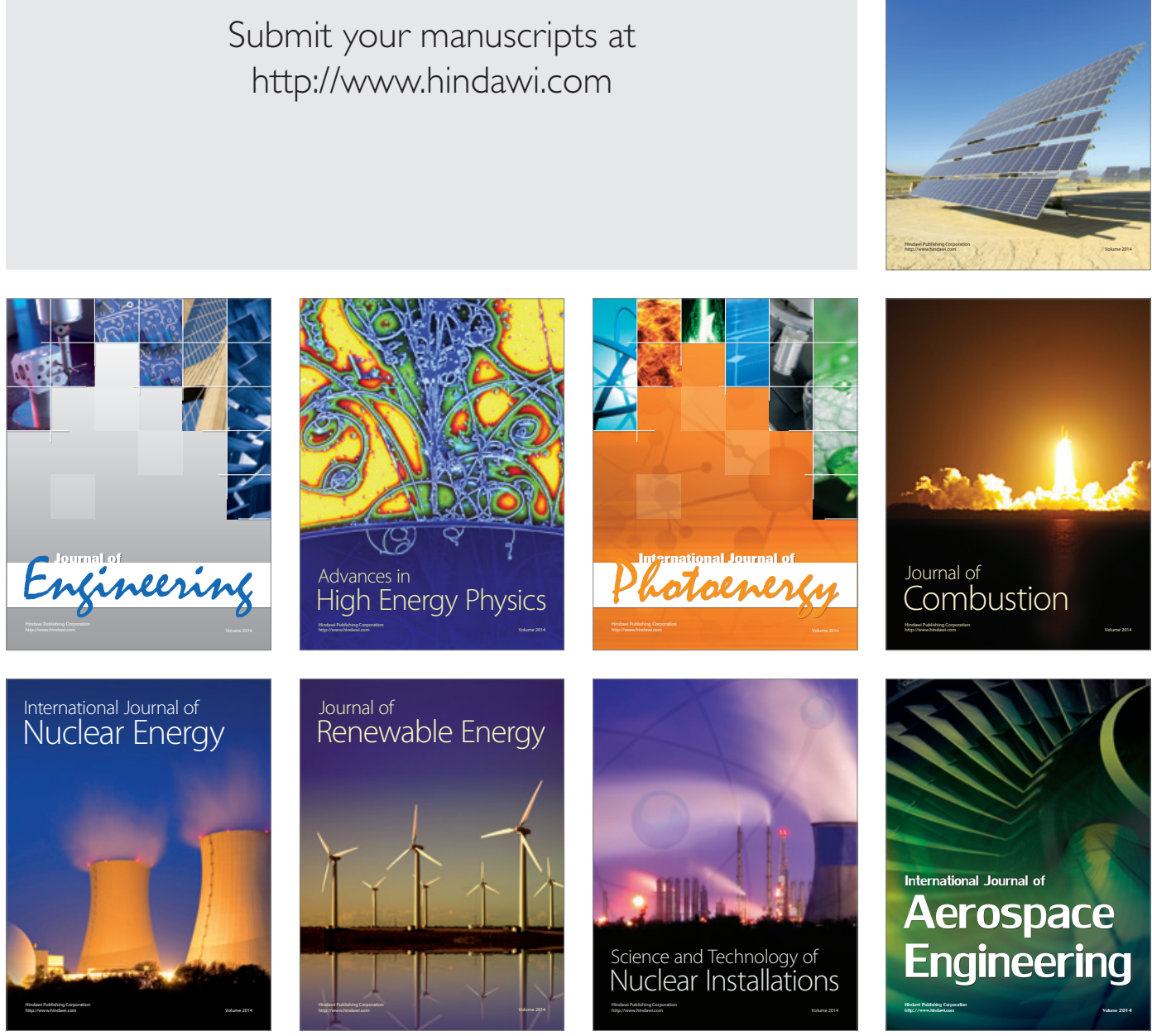